\title{
Prevalence of Coagulation Factors Deficiency among Young Adults in Saudi Arabia: A National Survey
}

\author{
${ }^{1}$ Department of Medicine, King Saud University Medical City, King \\ Saud University, Riyadh, Saudi Arabia \\ 2 Department of Genetics, King Faisal Specialist Hospital and \\ Research Centre, and College of Medicine, Alfaisal University, \\ Riyadh, Saudi Arabia \\ ${ }^{3}$ Research Center, King Faisal Specialist Hospital, Alfaisal University, \\ Riyadh, Saudi Arabia \\ ${ }^{4}$ Department of Pediatric, Taibah University, Medina, Saudi Arabia \\ ${ }^{5}$ College of Medical Applied Sciences, King Saud University, Riyadh, \\ Saudi Arabia \\ ${ }^{6}$ Department of Physiology, College of Medicine, King Saud \\ University, Riyadh, Saudi Arabia \\ ${ }^{7}$ Medina Maternity and Children Hospital, Medina, Saudi Arabia \\ 8 Department of Pathology, Imam Abdulrahman Bin Faisal University, \\ Dammam, Saudi Arabia
}

Khalid A. AlSaleh ${ }^{1}$ Nouf Al-Numair ${ }^{2}$ (1) Ayman AlSuliman $^{3}$ Mohammed Zolaly ${ }^{4}$ Abdul Majeed Albanyan ${ }^{5}$ Nouf AlOtaishan ${ }^{2}$ Esra Abudouleh ${ }^{5}$ Nervana Bayoumy ${ }^{6}$ Ahmad Tarawah ${ }^{7}$ Faisal AlZahrani ${ }^{8}$ Faisal AlAllaf $^{9}$ Abdul kareem AlMomen ${ }^{10}$ Raihan Sajid ${ }^{11}$ Tarek M. Owaidah ${ }^{12}$

\author{
Address for correspondence Tarek M. Owaidah, MBBS, Department \\ of Pathology and Laboratory Medicine, King Faisal Specialist Hospital \\ and Research Centre, Alfaisal University, P.O. Box 3354, Riyadh 11211, \\ Saudi Arabia (e-mail: towaidah@kfshrc.edu.sa). \\ ${ }^{9}$ Department of Molecular Medicine, Umm Al Qura University, \\ Makkah, Saudi Arabia \\ 10 Department of Pathology, College of Medicine, King Saud \\ University, Riyadh, Saudi Arabia \\ ${ }^{11}$ Department of Pathology, Alfaisal University, Riyadh, Saudi Arabia \\ 12 Department of Pathology and Laboratory Medicine, King Faisal \\ Specialist Hospital and Research Centre, Alfaisal University, Riyadh, \\ Saudi Arabia
}

\begin{abstract}
Keywords

- inherited

- bleeding

- coagulation

Introduction Inherited bleeding disorders vary in prevalence due to genetic disparity and ethnicity. Little is known about the prevalence of coagulation factor deficiency and bleeding disorders in middle-eastern population.

Methods Young Saudi adults with at least one positive bleeding symptom reported in semi-structured validated condensed MCMDM-1vWD questionnaire were tested for complete blood count, routine and special coagulation tests, serum ferritin level, and capillary zone electrophoresis. After initial testing, those with prolonged prothrombin time (PT) or activated prothrombin time (APTT) had further testing to evaluate coagulation factors level. Platelet function was tested through platelet function analyzer (PFA)-100, and multiplate aggregometer (MEA) on patients suspected of having platelet disorders.

Results Six-hundred-forty patients (male $=347,54.2 \%$ ) were included. A possible platelet function defect was diagnosed in three patients with one matching Glanzmann's thrombasthenia trait pattern, and one that of Bernard-Soulier trait pattern. One patient was diagnosed with von Willebrand disease. Deficiencies in coagulation factor levels were revealed as F-VIII in 14 (7.4\%), F-IX in 15 (7.6\%), F-II in two (3.3\%), F-V in 17 $(26.1 \%), F V I I$ in two $(3.1 \%)$, and F-X in one $(1.8 \%)$ of study subjects; low vWF activity $(<50 \%)$ was found in $14(8 \%)$. Abnormal values were found for various laboratory tests with prolongation of platelet function analyzer-epinephrine (PFA-EPI) in 11\%, PFA-ADP or arachidonic acid in $15.2 \%$, PT in 35.9\%, and APTT in 63.7\%. Five-hundred-seventy-six patients $(90 \%)$ had normal results in the coagulation factor assays and were
\end{abstract}

received

August 18, 2020 accepted after revision October 27, 2020
DOI https://doi.org/

10.1055/s-0040-1721500. ISSN 2512-9465.
(C) 2020 Georg Thieme Verlag KG Rüdigerstraße 14, 70469 Stuttgart, Germany
License terms

(c) (i) 
categorized as patients with bleeding of unknown cause (BUC). A diagnosis of a bleeding disorder was more frequently made in men than in women (38 vs. 26). Iron deficiency anemia was found in 18 (25\%) females positively associated with F-IX deficiency ( $p$-value 0.000$)$. Male gender $(73.3 \%, p=0.007)$ was independently associated with the diagnosis of coagulation factor deficiency.

Conclusion The current study reports a higher prevalence of coagulation factors deficiency in Saudi population than reported in the western population.

\section{Introduction}

Hereditary bleeding disorders are a vast group of hemostasis abnormalities resulting from problems in platelet number, adhesion, or aggregation; or deficiencies of coagulation factors, contact factors, fibrinogen (FIB), or connective tissue. Bleeding disorders are manifested as repeated provoked or spontaneous bleeding into joints, muscles, or mucocutaneous tissues depending upon the disease severity in each individual. ${ }^{1}$ The availability of advanced diagnostic and highly effective preventive and therapeutic measures may help maximize the benefits and minimize the risks of these disorders in diagnosing earlier than the development of complications. ${ }^{2}$ However, quantification of bleeding disorders based upon symptoms is a challenging task. Besides severe bleeding disorders, correct diagnosis is also necessary to decide on preventive measures for mild to moderate bleeding disorders (MBDs) including platelet function disorders, von Willebrand disease (vWD), and clotting factor deficiencies. ${ }^{3}$ Although MBDs do not lead to higher mortality rates, yet they present a potential motive for the intervention of hemostatic agents and blood products during and after surgical procedures to control bleeding or mild chronic bleeding. ${ }^{4}$ MBDs often do not distinctively manifest earlier in life, and may become evident only after significant hemostatic challenges. Diagnostic criteria are more inconsistent for MBDs than severe bleeding disorders and have not been addressed adequately. ${ }^{5}$ Bleeding of unknown cause (BUC), on the other hand, is a condition where MBDs cannot be associated with any genetic or hemostatic abnormality even after extensive investigation with contemporary techniques. ${ }^{6}$

Several studies reported prevalence of inherited bleeding disorders on a small scale in Saudi population; however, objective and focus of these studies revolved around vWD, hemophilia $\mathrm{A}$, hemophilia $\mathrm{B}$, and platelet disorders. ${ }^{7,8}$ Identification of cases based upon minimal symptoms may lead to overestimation of the prevalence of specific disease in population screening process. More stringent criteria in terms of accuracy and precision can be proposed to lessen the burden of a false-positive diagnosis.

Prothrombin time (PT) and activated prothrombin time (APTT) are the routinely performed coagulation tests with limited sensitivity to detect coagulation factor bleeding disorders. Platelet function analyzer (PFA)-100 is used for screening of primary hemostasis disorders with excellent sensitivity, yet its lack of specificity and predictive power of any particular disorder hampers its clinical utility as the sole testing system. Nonetheless, being a platelet functional test, it's a worthwhile addition when used in conjugation with other diagnostic and monitoring. ${ }^{9-11}$

The current study aimed to link symptoms-based evaluation with routine laboratory screening testing, complete blood picture with coagulation factors, and platelet aggregation studies to predict the prevalence of coagulation factor deficiencies and platelet disorder as a cause of bleeding symptoms.

\section{Methods}

\section{Study Design}

Our study is based on a large national epidemiological survey which we conducted in four major regions of KSA, and we are generating several substudies with different laboratory tests conducted as part of that survey. After the multicenter IRB approval, participants from both genders were selected randomly, and only young Saudi adults were included. A semi-structured validated condensed MCMDM-1vWD Bleeding Questionnaire, including questions related to different bleeding symptoms, was used. The results of that questionnaire are presented in another under-publication manuscript. Only those participants giving a positive response to any primary question were further sampled for various blood coagulation, platelet functions testing, ferritin, and hemoglobinopathy tests. The sample collection was conducted in all four regions, but all testing was performed at Center of Excellence Thrombosis and Hemostasis, King Saud University Medical City, Riyadh-except for PFA-100 (Siemens Healthcare Diagnostics, Malvern, Pennsylvania, United States) which was performed on site.

\section{Sample Collection}

Each of these participants had $10 \mathrm{~mL}$ of ethylenediaminetetraacetic acid (EDTA), $10 \mathrm{~mL}$ of citrated blood (at 3.2\%), and $5 \mathrm{~mL}$ of sodium heparin was collected. Complete blood count (CBC), serum ferritin level, and capillary zone electrophoresis were performed for each participant. $\mathrm{CBC}$ was tested on the same day from the EDTA samples using an automated SYSMEX XN-10 instrument (Sysmex Corporation, Kobe, Japan). Serum ferritin level was measured using an automated chemistry analyzer COBAS 601 (Roche Diagnostics, Basel, Switzerland). ABO blood grouping was performed via Diamed Gel card (Changsha Yingtai Instrument Co., Ltd).

All samples for coagulation tests including PT and APTT and other coagulation factors were separated within 2 to 4 hours from collection. These samples were centrifuged and plasma separated by standard techniques which were then 
transported in a frozen state to the central laboratory for testing (Center of Thrombosis and Hemostasis, King Saud University Medical City, Riyadh).

To maximize the study efficiency, a group of hematologists who are part of this study designed a stepwise approach based upon initial screening tests, including PT, APTT, and PFA-100. Those with prolonged PT alone were tested for extrinsic pathways (F-II, F-V, F-VII, and F-X), and those with prolonged APTT alone were tested for intrinsic pathways (F-XI, F-X, F-XI, and F-VIII), while those who were prolonged for APTT and PFA-100 were tested for vWF Ag and function. Participants who tested normal for APTT and prolonged for PFA-100 were tested for platelet aggregation. All coagulation tests were performed on STA R Max (Diagnostica Stago, Marseille, France). Those with possible platelet disorders were called back again seeking consent for another sample collection for platelet aggregation by multiplate aggregometer.

Since ours is a national survey consisting of different physical collection sites and because of the nature of coagulation tests, the investigators set stringent measures to ensure the quality and accuracy of the testing, and samples not meeting these measures were discarded.

\section{Statistical Analysis}

Descriptive statistics were computed as a baseline means, standard deviations, and minimum and maximum values for continuous variables. The inherited bleeding disorders were analyzed using laboratory results, including PFA, CBC, clotting factors, and ferritin. Chi-square test was used for comparing clotting factor with ferritin and hemoglobin against platelet disorder and clotting factor to confirm the severity of the factor deficiency or functional platelet disorder, which results in iron deficiency anemia (IDA). We used the software STATA v.13.0 (Stata Corp., College Station, Texas, United States) in our analysis. A statistical significance threshold of $p<0.05$ was adopted.

\section{Results}

Out of 848 young Saudi adults who affirmed to have at least one bleeding symptom, we performed laboratory testing on 640, where 347 (54.2\%) were males, and 293 (45.7\%) were females. Mean values of laboratory tests are summarized in - Table 1. For PFA test, EPI mean value was $135 \pm 35 \mathrm{sec}-$ onds $(n=534)$, ADP was $99.8 \pm 25.8$ seconds $(n=429)$, PT was $14.3 \pm 1.2$ seconds $(n=637)$, and APTT was $43.8 \pm 9$ seconds $(n=638)$. Abnormal values were found for laboratory PFA-EPI in $11 \%$, PFA-ADP in $15.2 \%$, PT in $35.9 \%$, and APTT in 63.7\%.

The mean values for coagulation factor-related tests were: FIB $266.7 \pm 64.4 \mathrm{IU} / \mathrm{mL}(n=9)$, F-II $93.2 \pm 25.5 \mathrm{IU} / \mathrm{mL}(n=61)$, F-V $68.8 \pm 41 \mathrm{IU} / \mathrm{mL}(n=65), \mathrm{F}-\mathrm{VII} 78.8 \pm 41 \mathrm{IU} / \mathrm{mL}(n=65), \mathrm{F}-$ VIII $101.4 \pm 48.7 \mathrm{IU} / \mathrm{mL}(n=187), F-I X \quad 104.9 \pm 37.9 \mathrm{IU} / \mathrm{mL}$ $(n=198), \mathrm{F}-\mathrm{X} 78.6 \pm 17.2 \mathrm{IU} / \mathrm{mL}(n=56), \mathrm{F}-\mathrm{XI} 108.3 \pm 44 \mathrm{IU} /$ $\mathrm{mL}(n=101)$, F-XIII $112.6 \pm 32.4 \mathrm{IU} / \mathrm{mL}(n=102)$. The mean values of $\mathrm{CBC}$ variables were HCT $41.3 \pm 5.2(n=628), \mathrm{MCV}$
$82.4 \pm 7.4(n=628)$, platelet $272.5 \pm 83.1(n=628)$, and MPV $9 \pm 1.1(n=627)$.

We applied $50 \%$ as a cut off for the diagnosis of coagulation factor deficiency. We found low F-VIII in 7.4\%, F-IX in 7.6\%, vWF activity in $8 \%$, F-II in 3.3\%, F-V in $26.1 \%$, FV-II in $3.1 \%$, and F-X in $1.8 \%$ study subjects. Of note, among abnormal factors level, 1 (0.5\%) was F-VIII + vWF Ag case (-Table 1). Genderbased factors deficiency is depicted in - Figs. 1 and 2, where eight females and seven males were deficient in F-VIII; four females and 11 males were deficient in F-IX ( $p$-value 0.007); one female and one male were deficient in F-II; seven females and 10 males had F-V deficiency, only one female was deficient in $\mathrm{F}-\mathrm{X}$, while five females and nine males were deficient in vWF act. The figures also show 576 (90\%) participants having bleeding symptoms, but normal results in the coagulation assays and so were categorized as patients with BUC.

Platelet function tests are summarized in - Table 2: arachidonic acid (ADP) $82.8 \pm 19.9 \quad(n=26)$, aspirin (ASP) $91.5 \pm 22.8(n=26)$, collagen (COL) $74.5 \pm 20.3(n=23)$, ristocetin (RISTO) $86.5 \pm 28(n=26)$, thrombin receptoractivating peptide (TRAP) $106.1 \pm 20(n=26)$.

For diagnosis of platelet dysfunction, we applied the known pattern of abnormal responses to different agonists like ADP, RISTO, COL, identifying three patients in our cohort with possible platelet dysfunction (PPD), one among which matched the pattern of Glanzmann's thrombasthenia with low COL and RISTO, and one matched the pattern of Bernard Soulier with abnormal RISTO. These cases were correlated with hemoglobin and platelet count without any significant association (-Table $\mathbf{3}$ ).

For hemoglobin and ferritin, we calculated gender-based mean values. For male participants, the mean ferritin value was $97.9 \pm 64.7(n=229)$ while mean hemoglobin was $44.5 \pm 55.8(n=341)$. For female participants, the mean ferritin value was $36.4 \pm 59.7(n=110)$, while hemoglobin was $33.8 \pm 44.8(n=287,-$ Table 4$)$.

The minimum and maximum values of variables indicate that the normal range exceeds in several cases. Therefore, we set out to analyze the proportion of test subjects with normal and abnormal ranges to identify the possible cause of bleeding. Correlation analysis between abnormal factor levels and IDA was done for both males and females. Out of male patients, low ferritin and low factor ranking was only observed in F-IX for one (2.3\%).

For PFA-based tests as well as PT and APTT, values above the maximum normal ranges were considered prolonged, and they might be indicators for bleeding. Comparison between APTT and PFA-EPI and PFA-ADP is summarized in -Table 4, indicating an insignificant relationship.

\section{Discussion}

Bleeding disorders are a cluster of inherited disorders with varying prevalence rates depending upon ethnicity. While most known inherited bleeding disorders are hemophilia A and $\mathrm{B}$, they are relatively rare. ${ }^{12}$ Most MBDs are often unrecognized as patients bleed only during stress periods, surgery, 
e460 Bleeding Disorders among Young Saudi Adults AlSaleh et al.

Table 1 Basic descriptive analysis for laboratory results

\begin{tabular}{|c|c|c|c|c|c|}
\hline Variables & $N$ & Mean + SD & Min & Max & Abnormal, $n$ (\%) \\
\hline PFA-EPI & 534 & $135 \pm 35$ & 51 & 300 & $60(11)$ \\
\hline PFA-ADP & 429 & $99.8 \pm 25.8$ & 37 & 300 & $67(15.2)$ \\
\hline PT & 637 & $14.3 \pm 1.2$ & 10.7 & 28.2 & $230(35.9)$ \\
\hline APTT & 638 & $43.8 \pm 9$ & 29.4 & 172 & $407 / 638(63.7)$ \\
\hline F-VIII & 187 & $101.4 \pm 48.7$ & 30 & 335 & $14 / 187(7.4)$ \\
\hline F-IX & 198 & $104.9 \pm 37.9$ & 30 & 200 & $15 / 198(7.6)$ \\
\hline F-XI & 101 & $108.3 \pm 44$ & 50 & 200 & - \\
\hline F-XIII & 102 & $112.6 \pm 32.4$ & 64 & 200 & - \\
\hline vWF Ag & 164 & $86.0 \pm 28.6$ & 34 & 178 & - \\
\hline FVIII + vWF & & & & & $1(0.5 \%)$ \\
\hline vWF Act & 173 & $78.9 \pm 26.7$ & 35 & 178 & $14(8)$ \\
\hline F-II & 61 & $93.2 \pm 25.5$ & 41 & 250 & $2(3.3)$ \\
\hline $\mathrm{F}-\mathrm{V}$ & 65 & $68.8 \pm 41$ & 30 & 361 & $17(26.1)$ \\
\hline F-VII & 65 & $78.8 \pm 41$ & 47 & 361 & $2(3.1)$ \\
\hline $\mathrm{F}-\mathrm{X}$ & 56 & $78.6 \pm 17.2$ & 30 & 149 & $1(1.8)$ \\
\hline FIB & 9 & $266.7 \pm 64.4$ & 201 & 409 & - \\
\hline $\mathrm{HCT}$ & 628 & $41.3 \pm 5.2$ & 22.4 & 56.5 & - \\
\hline MCV & 628 & $82.4 \pm 7.4$ & 52 & 107.7 & - \\
\hline Platelet & 628 & $272.5 \pm 83.1$ & 26 & 823 & - \\
\hline MPV & 627 & $9 \pm 1.1$ & 1.9 & 14.6 & - \\
\hline
\end{tabular}

Abbreviations: ADP, arachidonic acid; APTT, activated prothrombin time; EPI, epinephrine; FIB, fibrinogen; PFA, platelet function analyzer; PT, prothrombin time; SD, standard deviation; vWF, von Willebrand factor; HCT, hematocrit, MCV, mean corpuscular volume; MPV, mean platelet volume.

or medical procedures. ${ }^{12}$ In Saudi Arabia, other than case reports and case series, no large scale population-based screening studies have reported the prevalence of bleeding disorders. ${ }^{8,13,14}$ Arab population may have a higher prevalence of bleeding disorders than Western population, owing primarily to a higher consanguinity in Arab communities. ${ }^{15}$ The goal of the current study was to conduct the first populationscreening focused on bleeding disorders, correlating them with laboratory findings among young adults in Saudi Arabia.

In the current study, laboratory testing was performed for young Saudi adults who affirmed to have at least one bleeding

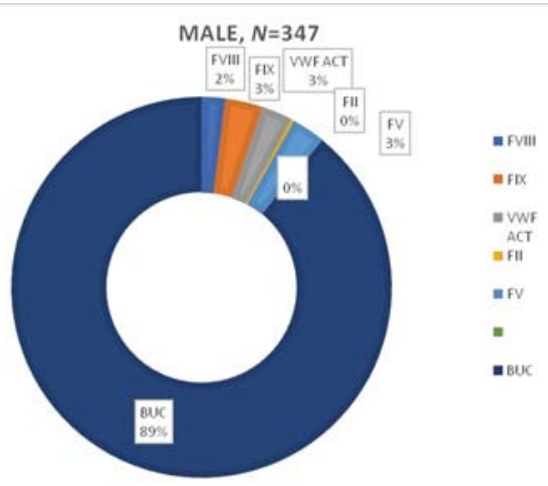

Fig. 1 Factors deficiency in males. symptom. The minimum and maximum values of variables indicated that the normal range was exceeded in several cases while ABO-corrected ${ }^{16}$ vWFAg in four (2.4\%) study subjects. Ahmed et al previously reported one factor VII deficiency, one factor X deficiency in 34 cases of inherited bleeding disorders from Eastern Province of Saudi Arabia, along with five unidentified platelet function disorders. ${ }^{13}$ In the current study, three patients had PPD, one among which had a matching pattern of Glanzmann's thrombasthenia. ${ }^{17}$ Al-Sharif et al reported clinical phenotype of around 20 patients with factor XIII deficiency

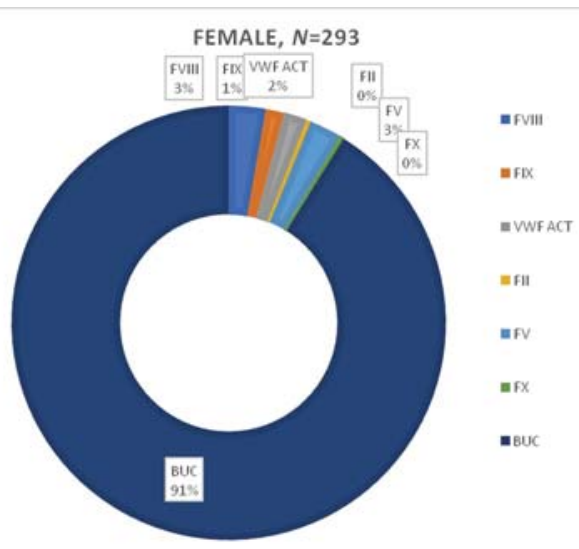

Fig. 2 Factor deficiency in females. 
Table 2 Platelet function test's description

\begin{tabular}{|l|l|l|l|l|l|}
\hline Variables & N & Mean + SD & Min & Max & Abnormal $\boldsymbol{n}(\%)$ \\
\hline ADP & 26 & $82.8 \pm 19.9$ & 36 & 117 & $1(3.8)$ \\
\hline ASP & 26 & $91.5 \pm 22.8$ & 46 & 145 & $6(23.1)$ \\
\hline COL & 23 & $74.5 \pm 20.3$ & 40 & 127 & $5(19.2)$ \\
\hline RISTO & 26 & $86.5 \pm 28$ & 42 & 149 & $7(26.9)$ \\
\hline TRAP & 26 & $106.1 \pm 20$ & 65 & 138 & $6(23.1)$ \\
\hline
\end{tabular}

Abbreviations: ADP, arachidonic acid; ASP, aspirin; COL, collagen; EPI, epinephrine; RISTO, ristocetin; SD, standard deviation; TRAP, thrombin receptor-activating peptide.

Table 3 Comparison of PFA-EPI with PFA-ADP for normal and prolonged values

\begin{tabular}{|l|l|l|l|}
\hline PFA-ADP & $\begin{array}{l}\text { PFA-EPI } \\
\text { normal }\end{array}$ & $\begin{array}{l}\text { PFA-EPI } \\
\text { prolonged }\end{array}$ & Total \\
\hline Normal & $331(93.5 \%)$ & $39(60.9 \%)$ & $370(88.5 \%)$ \\
\hline Prolonged & $23(6.5 \%)$ & $25(39.1 \%)$ & $48(11.5 \%)$ \\
\hline
\end{tabular}

Abbreviations: ADP, arachidonic acid; EPI, epinephrine; PFA, platelet function analyzer.

Note: $p$-Value $<0.001$.

Table 4 Ferritin and Hgb per gender

\begin{tabular}{|l|l|l|l|l|l|}
\hline Variables & $n$ & Mean + SD & Min & Max & IDA \\
\hline & \multicolumn{5}{|c|}{ Males } \\
\hline Ferritin & 229 & $97.9 \pm 64.7$ & 5.5 & 543 & 0 \\
\hline Hgb & 341 & $44.5 \pm 55.8$ & 7.6 & 168 & \\
\hline & \multicolumn{3}{|c|}{ Females } & & \\
\hline Ferritin & 110 & $36.4 \pm 59.7$ & 1.65 & 393 & $\begin{array}{l}18 \\
(25 \%)\end{array}$ \\
\hline Hgb & 287 & $33.8 \pm 44.8$ & 6.8 & 153 & \\
\hline
\end{tabular}

Abbreviations: Hgb, hemoglobin; IDA, iron deficiency anemia.

in Riyadh region. ${ }^{14}$ In an 8-year retrospective analysis of 168 inherited bleeding disorder patients by Al-Fawaz et al, patients with factor XI deficiency: four with factors V and VIII deficiency, and one with factor VII deficiency were reported, while vWD was stated as the second most common cause of hereditary bleeding disorder. ${ }^{7}$ Sadler et al in his review, reported vWD to be one of the most common inherited bleeding diseases, with a conservative prevalence of 100 per million persons, putting the total at 580,000 persons. ${ }^{18}$ Over the span of 25 years, Madkhali et al found 38 patients with rare clotting factor disorders; one had afibrinogenemia deficiency, two with F-II, three with F-V, six with F-VII, one with F-X, nine with F-XI, six with F-XII, and nine patients had F-XIII deficiency, while one patient had combined FV and FVIII deficiency. ${ }^{19}$ Quiroga et al reported diagnostic efficacy of laboratory testing in vWD patients with hereditary mucocutaneous bleeding as $40.4 \%$, and identified $17 \%$ patients with vWD, ${ }^{20}$ while Friberg et al reported the presence of at least one bleeding symptom in $73 \%$ participants among 1,410 surveyed girls. ${ }^{21}$ The frequency of coagulation factor abnormalities reported in the current study is greater than all these previous studies due to a larger sample size, which also indicates the greater statistical power of our study. Gebhart et al found in his hospital-based study that 25 to $50 \%$ with MBDs could be diagnosed with a specific cause, whereas in our population-based study only $10 \%$ MBDs could be diagnosed with deficiencies, remaining $90 \%$ were labeled as BUC. ${ }^{6}$

In chi-square test of independence, a significant association was found in the current study between diagnosis and PFA-EPI and PFA-ADP results. Conflicting results have been reported previously regarding platelet function evaluated by PFA-100. A positive relationship between the extent of bleeding and platelet dysfunction was reported by Ostrowsky et al, ${ }^{22}$ and Raman and Silverman, ${ }^{23}$ while Fattorutto et al, ${ }^{24}$ and Forestier et $\mathrm{al}^{25}$ reported inconsistency in this association.

To explore the association of $\mathrm{Hgb}$ and ferritin with abnormalities in laboratory testing variables, the laboratory testing for coagulation factors and vWD Ag for subjects with low $\mathrm{Hgb}$ and abnormal Ferritin was compared. We found a correlation of abnormal ferritin level with factor $\mathrm{X}$ deficiency in males and with vWD Ag in females and low Hgb with FVIII abnormalities in males and females, thus, depicting the predictive nature of $\mathrm{Hgb}$ and ferritin for bleeding disorder identification. Low $\mathrm{Hgb}$ and/or ferritin are weak predictors of bleeding disorders at best, because there are various other causes of anemia and iron deficiency.

\section{Conclusion}

The current study reports the prevalence of coagulation factor deficiency based upon never been done before population laboratory testing, which could explain the higher prevalence of bleeding disorders in our region. Coagulation factor deficiencies and vWF Ag and activity have been found associated with IDA in Saudi patients suffering from bleeding disorders. We plan on future studies based on molecular profiling.

\section{Authors' Contributions}

K.A.S. and T.M.O. designed and developed the study. Both authors were responsible for contents and authenticity. N.A.-N., A.S., M.Z., A.A. oversaw data collection and data entry. N.A.O., E.A., N.B., and A.T. performed final review of data and analysis. F.A.Z., F.A.A., A.A.A., and R.S. were responsible for direction of the study team and facilitation of the project plan. 


\section{Note}

The study was approved by the Institutional Review Board of King Faisal Specialist Hospital and Research Center, Kingdom of Saudi Arabia.

\section{Funding}

This study was supported by KACST (408-34) RAC KFSHRC (2130036).

\section{Conflict of Interest}

None declared.

\section{References}

1 Peyvandi F, Palla R, Menegatti M, Mannucci PM. Introduction: rare bleeding disorders: general aspects of clinical features, diagnosis, and management. Semin Thromb Hemostasis 2009;35(04):349-355

2 Boender J, Kruip MJ, Leebeek FW. A diagnostic approach to mild bleeding disorders. J Thromb Haemost 2016;14(08):1507-1516

3 Greaves M, Watson HG. Approach to the diagnosis and management of mild bleeding disorders. J Thromb Haemost 2007;5 (Suppl 1):167-174

4 Hayward CP. Diagnosis and management of mild bleeding disorders. Hematology (Am Soc Hematol Educ Program) 2005;2005 (01):423-428

5 Mezzano D, Quiroga T. Diagnostic challenges of inherited mild bleeding disorders: a bait for poorly explored clinical and basic research. J Thromb Haemost 2019;17(02):257-270

6 Gebhart J, Hofer S, Panzer S, et al. High proportion of patients with bleeding of unknown cause in persons with a mild-to-moderate bleeding tendency: results from the Vienna Bleeding Biobank (VIBB). Haemophilia 2018;24(03):405-413

7 Al-Fawaz IM, Gader AM, Bahakim HM, Al-Mohareb F, Al-Momen AK, Harakati MS. Hereditary bleeding disorders in Riyadh, Saudi Arabia. Ann Saudi Med 1996;16(03):257-261

8 El-Bostany EA, Omer N, Salama EE, El-Ghoroury EA, Al-Jaouni SK. The spectrum of inherited bleeding disorders in pediatrics. Blood Coagul Fibrinolysis 2008;19(08):771-775

9 Angiolillo D, Levy J, Schneider D, Jennings L. The Role of Platelet Function Testing in Improving Clinical Outcomes. The Medical Roundtable Cardiovascular Edition; 2020

10 Favaloro EJ. Clinical utility of the PFA-100. Semin Thromb Hemostasis 2008;34:709-733

11 Paniccia R, Priora R, Liotta AA, Abbate R. Platelet function tests: a comparative review. Vasc Health Risk Manag 2015; 11:133-148
12 Owaidah T, Saleh M, Alzahrani H, et al. Prevalence of bleeding symptoms among adolescents and young adults in the capital city of Saudi Arabia. Adv Hematol 2018;2018:1858241

13 Ahmed MA, Al-Sohaibani MO, Al-Mohaya SA, Sumer T, Al-Sheikh $\mathrm{EH}$, Knox-Macaulay H. Inherited bleeding disorders in the Eastern Province of Saudi Arabia. Acta Haematol 1988;79(04): 202-206

14 Al-Sharif FZ, Aljurf MD, Al-Momen AM, et al. Clinical and laboratory features of congenital factor XIII deficiency. Saudi Med J 2002;23(05):552-554

15 Hamamy H. Consanguineous marriages: preconception consultation in primary health care settings. J Community Genet 2012;3 (03):185-192

16 Alharbi A, Hassan SB, Al-Momen A-K, et al. Influence of ABO blood group on von Willebrand factor tests in healthy Saudi blood donors. Blood Coagul Fibrinolysis 2018;29(02):211-215

17 Albanyan A, Al-Musa A, AlNounou R, et al. Diagnosis of Glanzmann thrombasthenia by whole blood impedance analyzer (MEA) vs. light transmission aggregometry. Int J Lab Hematol 2015;37 (04):503-508

18 Sadler JE, Mannucci PM, Berntorp E, et al. Impact, diagnosis and treatment of von Willebrand disease. Thromb Haemost 2000;84 (02):160-174

19 Madkhali I, Owaidah T, Riash MA, Naser R, Al-Zahrani H. Rare inherited clotting factor deficiencies: a single-center experience. J Appl Hematol 2012;3(01):36-38

20 Quiroga T, Goycoolea M, Panes O, et al. High prevalence of bleeders of unknown cause among patients with inherited mucocutaneous bleeding. A prospective study of 280 patients and 299 controls. Haematologica 2007;92(03):357-365

21 Friberg B, Ornö AK, Lindgren A, Lethagen S. Bleeding disorders among young women: a population-based prevalence study. Acta Obstet Gynecol Scand 2006;85(02):200-206

22 Ostrowsky J, Foes J, Warchol M, Tsarovsky G, Blay J. Plateletworks platelet function test compared to the thromboelastograph for prediction of postoperative outcomes. J Extra Corpor Technol 2004;36(02):149-152

23 Raman S, Silverman NA. Clinical utility of the platelet function analyzer (PFA-100) in cardiothoracic procedures involving extracorporeal circulation. J Thorac Cardiovasc Surg 2001;122(01): 190-191

24 Fattorutto M, Pradier O, Schmartz D, Ickx B, Barvais L. Does the platelet function analyser (PFA-100) predict blood loss after cardiopulmonary bypass? Br J Anaesth 2003;90(05):692-693

25 Forestier F, Coiffic A, Mouton C, Ekouevi D, Chêne G, Janvier G. Platelet function point-of-care tests in post-bypass cardiac surgery: are they relevant? $\mathrm{Br}$ J Anaesth 2002;89 (05):715-721 